\title{
Reactivity of 1-alkyl-2-(bromomethyl)aziridines towards n-butyllithium
}

\author{
Matthias D’hooghe and Norbert De Kimpe* \\ Department of Organic Chemistry, Faculty of Bioscience Engineering, Ghent University, \\ Coupure Links 653, B-9000 Ghent, Belgium \\ E-mail: norbert.dekimpe@UGent.be
}

\section{Dedicated to Prof. Alain Krief on the occasion of his 65th birthday}

\begin{abstract}
The reactivity of 1-alkyl-2-(bromomethyl)aziridines with regard to $n$-butyllithium has been evaluated for the first time, resulting in a variety of reaction products due to competitive reaction pathways. The main components in these mixtures are 1-alkyl-2-(N-alkyl-Nallylaminomethyl)aziridines (11-36\%), 1-alkyl-2-pentylaziridines (13-26\%), $N$-alkyl- $N$-allyl- $N$ butylamines (1-24\%), $N$-alkyl- $N$-pentylamines (4-12\%) and $N$-alkyl- $N$-allylamines (1-7\%). A few of these components were isolated by means of preparative gas chromatography. The structural identity of some other constituents has been proven by independent syntheses.
\end{abstract}

Keywords: 2-(Bromomethyl)aziridines, ring opening, n-butyllitium

\section{Introduction}

Aziridines are versatile building blocks for the synthesis of a large variety of ring opened and ring expanded amines due to the inherent reactivity of these compounds, ${ }^{1}$ an effect undoubtedly resulting from the necessary compression of bond angles in the three-membered ring. Although many reports on the utility of substituted aziridines in organic synthesis are available, 1-alkyl-2(bromomethyl)aziridines comprise a peculiar and rather unknown class of non-activated aziridine derivatives with high synthetic potential due to three different electrophilic carbon atoms in their structure. $^{2}$

As reported before, 1-alkyl-2-(bromomethyl)aziridines are suitable synthetic equivalents for the aziridinylmethyl cation, providing an easy access to 1,2-dialkylaziridines such as 2-ethyl-, 2pentyl- and 2-(phenylmethyl)aziridines upon treatment with the appropriate lithium dialkylcuprate reagent. ${ }^{3}$ A similar reactivity of 1-alkyl-2-(bromomethyl)aziridines has been observed in reaction with oxygen-centered nucleophiles such as alkoxides, phenoxides and 
carboxylates, affording the corresponding 2-substituted aziridines in a clean and straightforward reaction. ${ }^{4}$ Very recently, the reaction of 2-(bromomethyl)aziridines with methyllithium has been reported to afford 1-alkyl-2-( $N$-alkyl- $N$-ethylaminomethyl)aziridines through a highly unusual reaction pathway. ${ }^{5}$ However, up to now, the reactivity of 1-alkyl-2-(bromomethyl)aziridines with regard to $n$-butyllithium, a frequently used reagent in organic chemistry, ${ }^{6}$ has not been investigated yet, and this will be discussed in the present report.

\section{Results and Discussion}

Treatment of 1-neopentyl- and 1-isobutyl-2-(bromomethyl)aziridines 1a,b, prepared according to literature procedures, ${ }^{7}$ with 1.5 equiv of $n$-butyllithium in dry diethyl ether or THF under nitrogen atmosphere resulted in complex reaction mixtures. In these mixtures, the presence of aziridines and allylic groups could be derived from ${ }^{1} \mathrm{H}$ NMR spectra $(\delta$ 1.2-1.6 and 5.0-6.0 ppm respectively, $\mathrm{CDCl}_{3}$ ). The assignment of the molecular structures of the major constituents 2-6 has been established by detailed mass spectroscopic analysis based on the molecular ion and the fragmentation pattern of each compound, and acknowledged by comparison with data obtained from independent syntheses of some examples. The main components are 1-alkyl-2-( $N$-alkyl- $N$ allylaminomethyl)aziridines 2a,b (11-36\%) and 1-alkyl-2-pentylaziridines 3a,b (13-26\%). Starting from 1-neopentylaziridine 1a, $N$-allyl- $N$-butyl- $N$-neopentylamine $4 \mathbf{a}$ is also present in a substantial amount (13-24\%), whereas for the 1-isobutyl derivative the corresponding $N$-allyl- $N$ butyl- $N$-isobutylamine $\mathbf{4 b}$ is only a minor constituent (1-5\%). Furthermore, $N$-alkyl- $N$ pentylamines 5a,b (4-12\%) and $N$-alkyl- $N$-allylamines 6a,b (1-7\%) were identified, again in a larger amount starting from 1-neopentylaziridine 1a when compared to 1-isobutylaziridine $\mathbf{1 b}$. Obviously, competitive reaction pathways give rise to different reaction products upon treatment of 1-alkyl-2-(bromomethyl)aziridines 1 with $n$-butyllithium, in contrast with the previously observed reactivity of these aziridines 1 towards other organometallic reagents such as lithium dialkylcuprates, in which displacement of the bromo atom resulted in the corresponding 2substituted aziridines as the sole reaction products.
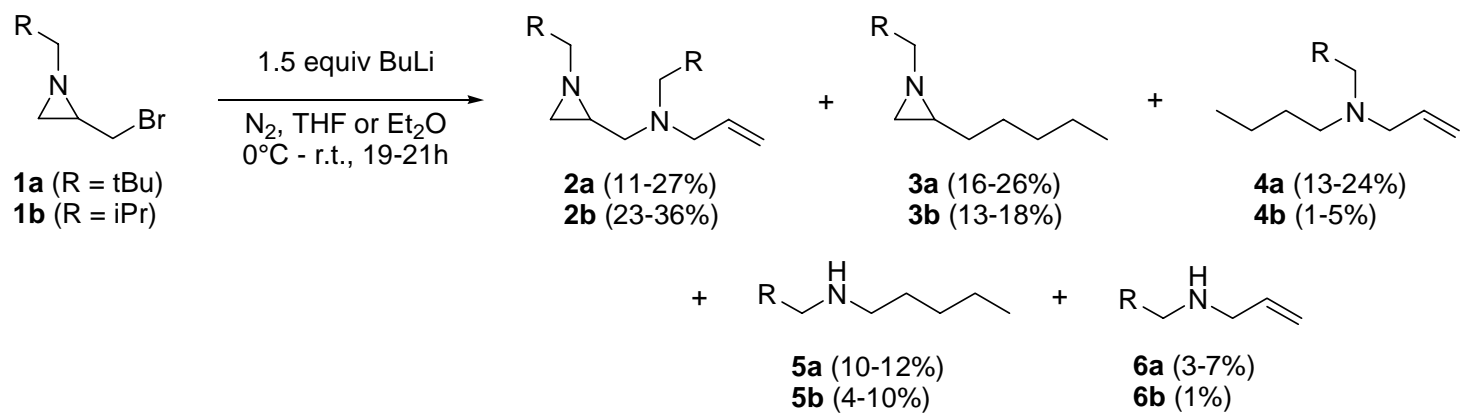

\section{Scheme 1}


The formation of 2-(aminomethyl)aziridines 2 can be rationalized considering a halophilic reaction by the butyl anion onto the bromo atom, followed by ring opening of the intermediate aziridinylmethyl anion towards $\mathrm{N}$-allyl lithium amides 7 and liberation of butyl bromide (Scheme 2). These lithium amides 7 can act as nucleophiles in a displacement reaction with unreacted 2(bromomethyl)aziridine 1, affording 2-(aminomethyl)aziridines 2. If no electrophilic substrate $\mathbf{1}$ is available anymore, lithium amides 7 will be protonated during workup resulting in allylamines 6 (Scheme 2).

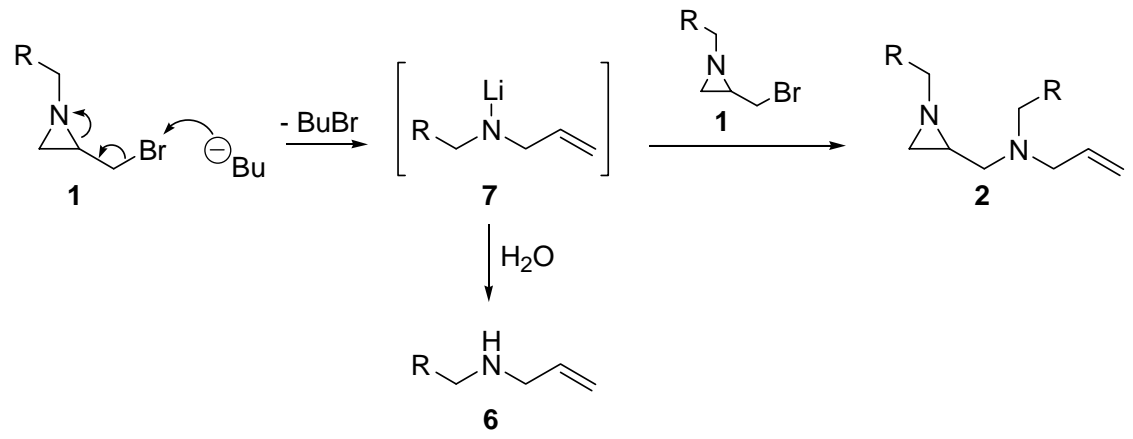

\section{Scheme 2}

To prove the presence of 2-(aminomethyl)aziridines 2, 2-(bromomethyl)aziridines $\mathbf{1 a}$ and $\mathbf{1 b}$ were treated with lithium $N$-allyl- $N$-neopentylamide and lithium $N$-allyl- $N$-isobutylamide in THF for 18 to 20 hours at room temperature under nitrogen atmosphere, furnishing the desired 2- $(N$ allylaminomethyl)aziridines 2, which were purified by means of column chromatography yielding $40 \%$ of $\mathbf{2 a}$ and $22 \%$ of $\mathbf{2} \mathbf{b}$ (Scheme 3). Comparison of retention times and mass spectra confirmed the presence of the aforementioned 2-( $N$-allylaminomethyl)aziridines 2 in the reaction mixtures obtained after treatment of aziridines $\mathbf{1}$ with BuLi. $N$-Allyl- $N$-neopentylamine $\mathbf{6 a}$ and $N$-allyl- $N$-isobutylamine $\mathbf{6 b}$ were prepared by imination of 2,2-dimethylpropanal (pivaldehyde) 8a and isobutyraldehyde $\mathbf{8 b}$ with allyl amine and magnesium sulfate in $\mathrm{CH}_{2} \mathrm{Cl}_{2}$ (96-98\%), followed by a reduction with $\mathrm{NaBH}_{4}$ in methanol (90-92\%, Scheme 4). Only one example of a 2( $N$-allylaminomethyl)aziridine could be found in the literature, i.e. 1-benzyl-2-( $N$-allyl- $N$ (benzyl)aminomethyl)aziridine. ${ }^{8}$
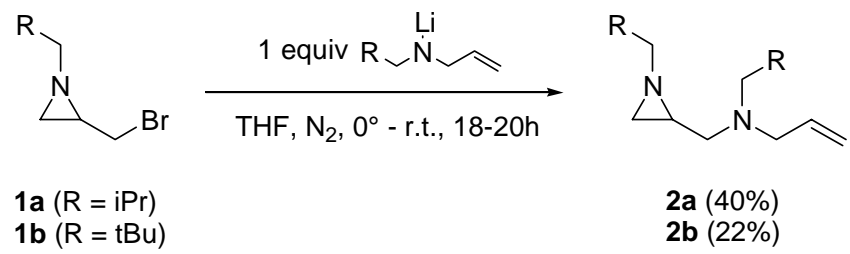

\section{Scheme 3}




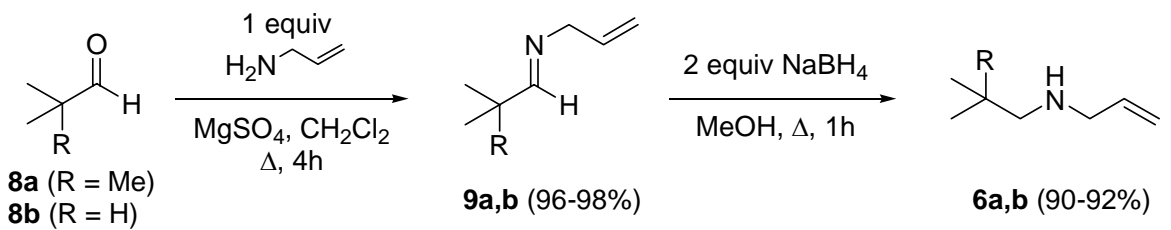

\section{Scheme 4}

The formation of 2-pentylaziridines $\mathbf{3}$ in the reaction mixtures obtained after treatment of aziridines 1 with $\mathrm{BuLi}$ is the result of a nucleophilic displacement of the bromo atom of aziridines 1 by a butyl anion, in accordance with the aziridinylmethyl cation equivalency of the latter compounds. Allylamines $\mathbf{4}$ can arise from a nucleophilic attack of lithium amides $\mathbf{7}$ onto in situ liberated butyl bromide (Scheme 5).

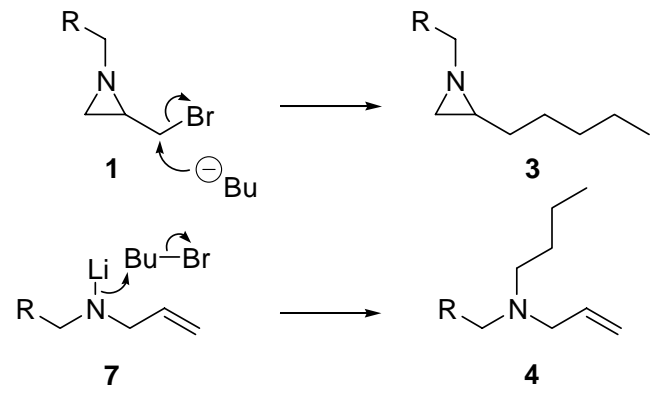

\section{Scheme 5}

A representative example of constituents $\mathbf{3}$ in the reaction mixtures has been prepared by an independent synthesis in order to prove their presence. 2-Pentylaziridine $\mathbf{3 b}$ was prepared by treatment of aziridine $\mathbf{1 b}$ with 1.5 equivalents of lithium dibutylcuprate in diethyl ether for 4 hours at room temperature (Scheme 6). ${ }^{3}$ Comparison of the retention time and mass spectra confirmed the presence of these compounds in the reaction mixtures obtained after treatment of 2-(bromomethyl)aziridines 1 with $n$-butyllithium.

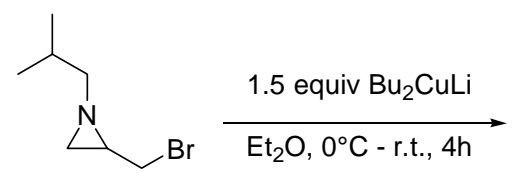

$1 \mathrm{~b}$

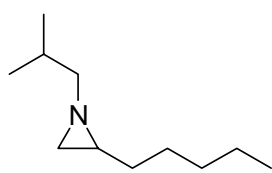

3b $(63 \%)$

\section{Scheme 6}

A plausible explanation for the formation of secondary amines 5 upon treatment of aziridines 1 with BuLi is depicted in Scheme 7, in which the substrate 1 undergoes a $S_{N} 2$ '-type substitution, followed by conversion of the resulting enamines $\mathbf{1 0}$ into lithium amides $\mathbf{1 1}$. The nucleophilic 
attack of a butyl carbanion at the unsubstituted aziridine carbon atom of aziridines $\mathbf{1}$ towards $\mathrm{N}$ alkyl- $N$-pentyl- $N$-vinylamines $\mathbf{1 0}$ via a $\mathrm{S}_{\mathrm{N}} 2$ '-type reaction is not unlikely, since also cyclopropanes behave in some respects like double-bond compounds due to the so called bent bonds. ${ }^{9}$ Small vinylamines are known to be unstable, ${ }^{10}$ and the thus quite labile enamines $\mathbf{1 0}$ can undergo deprotonation by the excess of butyllithium with expulsion of acetylene, resulting in $\mathrm{N}$ ethyl lithium amides 11. A similar, although far more selective, reactivity has been reported very recently, in which an analogous transformation was described upon treatment of 2(bromomethyl)aziridines with methyllithium, resulting in 1-alkyl-2-( $N$-alkyl- $N$ ethylaminomethyl)aziridines upon liberation of acetylene. ${ }^{5}$ Finally, neutralization of the lithium amides $\mathbf{1 1}$ during workup afforded amines $\mathbf{5}$.

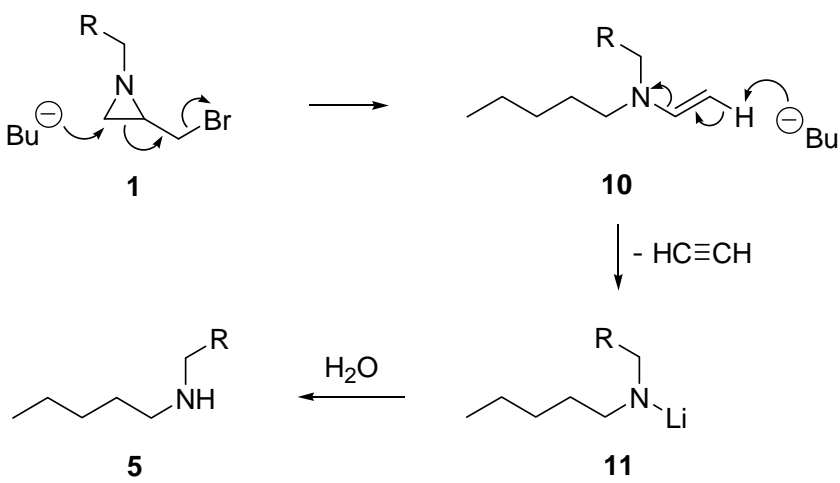

\section{Scheme 7}

In conclusion, treatment of 1-alkyl-2-(bromomethyl)aziridines with $n$-butyllithium results in the formation of a set of reaction products due to competitive reaction pathways in contrast with other more selective organometallic reagents such as methyllithium and organocuprates. The main components in these reaction mixtures are 1-alkyl-2-( $N$-alkyl- $N$ allylaminomethyl)aziridines (11-36\%), 1-alkyl-2-pentylaziridines (13-26\%), $N$-alkyl- $N$-allyl- $N$ butylamines (1-24\%), $N$-alkyl- $N$-pentylamines (4-12\%) and $N$-alkyl- $N$-allylamines (1-7\%), besides some other unidentified products in small quantities.

\section{Experimental Section}

General Procedures. ${ }^{1} \mathrm{H}$ NMR spectra were recorded at $270 \mathrm{MHz}$ (JEOL JNM-EX 270) or at $300 \mathrm{MHz}$ (JEOL ECLIPSE+) with $\mathrm{CDCl}_{3}$ as solvent and tetramethylsilane as internal standard. ${ }^{13} \mathrm{C}$ NMR spectra were recorded at $68 \mathrm{MHz}$ (JEOL JNM-EX 270) with $\mathrm{CDCl}_{3}$ as solvent. Mass spectra were obtained with a mass spectrometer (VARIAN MAT 112, $70 \mathrm{eV}$ using a GC-MS coupling (RSL 200, 20 m glass capillary column, i.d. $0.53 \mathrm{~mm}$, He carrier gas). IR spectra were measured with a Spectrum One FT-IR spectrophotometer. Preparative gas chromatography was performed using a Delsi Intersmat IGC 120 ML. Diethyl ether and tetrahydrofuran were distilled 
over sodium benzophenone ketyl, dichloromethane was distilled over calcium hydride. Other solvents were used as received from the supplier.

Treatment of 1-alkyl-2-(bromomethyl)aziridines 1 with $\boldsymbol{n}$-butyllithium. General procedure. To an ice-cooled solution of 2-(bromomethyl)aziridine 1 (5 mmol) in THF (10 mL) was added dropwise $n$-BuLi (3 mL, 1.5 equiv, 2.5M in hexane) via a syringe under nitrogen atmosphere. The resulting solution was further stirred at room temperature for 19-21 hours under nitrogen atmosphere. Workup was carried out by pouring the reaction mixture in an aqueous sodium hydroxide solution $\left(10 \mathrm{~mL}, 0.5 \mathrm{M}\right.$ in $\left.\mathrm{H}_{2} \mathrm{O}\right)$, followed by extraction with diethyl ether $(2 \times 10 \mathrm{~mL}$, $1 \times 5 \mathrm{~mL}$ ). After drying of the organic phase over $\mathrm{K}_{2} \mathrm{CO}_{3}$ and filtration of the drying agent, the solvent was removed in vacuo, and the resulting oil was analyzed by means of GC-MS or preparative gas chromatography.

Synthesis of 2-(N-allylaminomethyl)aziridines 2 . To an ice-cooled solution of $N$-allyl- $N$ neopentylamine 6a or $N$-allyl- $N$-isobutylamine $\mathbf{6 b}(5 \mathrm{mmol})$ in dry diethyl ether or THF (5 mL) was added dropwise $n$-BuLi ( $2 \mathrm{~mL}, 1$ equiv, $2.5 \mathrm{M}$ in hexane) via a syringe under nitrogen atmosphere. After stirring for 1 hour at $0^{\circ} \mathrm{C}$, a solution of 2-(bromomethyl)aziridine 1a,b (1 equiv) in THF ( $5 \mathrm{~mL}$ ) was added via a syringe at $0^{\circ} \mathrm{C}$. The resulting solution was further stirred at room temperature for 18-20 hours under nitrogen atmosphere. Workup was carried out by pouring the reaction mixture in an aqueous sodium hydroxide solution (10 $\mathrm{mL}, 0.5 \mathrm{M})$, followed by extraction with diethyl ether $(2 \times 10 \mathrm{~mL}, 1 \times 5 \mathrm{~mL})$. After drying of the organic phase with $\mathrm{K}_{2} \mathrm{CO}_{3}$ and filtration of the drying agent, the solvent was removed in vacuo, and the resulting aziridines 2 were purified by means of column chromatography on silica gel (Hexane/EtOAc 1/1).

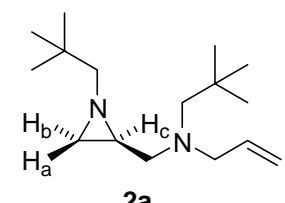

$2 a$

1-(2,2-Dimethylpropyl)-2-( $N$-allyl- $N$-(2,2-dimethylpropyl)aminomethyl)aziridine

(2a). Light-yellow oil. ${ }^{1} \mathrm{H}$ NMR (300 MHz, $\left.\mathrm{CDCl}_{3}\right): \delta 0.87$ and $0.95\left(18 \mathrm{H}, 2 \times \mathrm{s}, 2 \times\left(\mathrm{CH}_{3}\right)_{3}\right) ; 1.22(1 \mathrm{H}$, d, J=6.3 Hz, Hb); 1.38-1.46 (1H, m, $\left.\mathrm{H}_{\mathrm{c}}\right) ; 1.57\left(1 \mathrm{H}, \mathrm{d}, \mathrm{J}=3.6 \mathrm{~Hz}, \mathrm{H}_{\mathrm{a}}\right) ; 1.93$ and $2.13(2 \mathrm{H}, 2 \times \mathrm{d}$, $\left.\mathrm{J}=11.8 \mathrm{~Hz}, \mathrm{CH}_{\mathrm{C}} \mathrm{NCH}_{2} \mathrm{tBu}\right) ; 2.23\left(2 \mathrm{H}, \mathrm{s}, \mathrm{CH}_{2}=\mathrm{CHCH}_{2} \mathrm{NCH}_{2} \mathrm{tBu}\right) ; 2.32$ and $2.76(2 \mathrm{H}, 2 \times \mathrm{d} \times \mathrm{d}$, $\left.\mathrm{J}=13.4,6.7,4.7 \mathrm{~Hz},\left(\mathrm{H}_{\mathrm{a}} \mathrm{CH}_{\mathrm{b}}\right) \mathrm{CH}_{\mathrm{c}} \mathrm{CH}_{2} \mathrm{~N}\right)$; $3.19\left(2 \mathrm{H}, \mathrm{d} \times \mathrm{t}, \mathrm{J}=6.3,1.4 \mathrm{~Hz}, \mathrm{NCH}_{2} \mathrm{CH}=\mathrm{CH}_{2}\right)$; 5.05$5.17\left(2 \mathrm{H}, \mathrm{m}, \mathrm{CH}=\mathrm{CH}_{2}\right) ; 5.80-5.93\left(1 \mathrm{H}, \mathrm{m}, \mathrm{CH}=\mathrm{CH}_{2}\right) .{ }^{13} \mathrm{C} \mathrm{NMR}\left(68 \mathrm{MHz}, \mathrm{CDCl}_{3}\right): \delta 28.1$ and $28.2\left(2 \times\left(\mathrm{CH}_{3}\right)_{3} \mathrm{C}\right) ; 32.6$ and $33.2\left(2 \times\left(\mathrm{CH}_{3}\right)_{3} \mathrm{C}\right) ; 33.9\left(\mathrm{H}_{\mathrm{a}} \mathrm{CH}_{\mathrm{b}}\right) ; 38.5\left(\mathrm{CH}_{\mathrm{c}}\right) ; 59.4$ and 60.0 $\left(\left(\mathrm{H}_{\mathrm{a}} \mathrm{CH}_{\mathrm{b}}\right) \mathrm{CH}_{\mathrm{c}} \mathrm{CH}_{2} \mathrm{~N}\right.$ and $\left.\mathrm{NCH}_{2} \mathrm{CH}=\mathrm{CH}_{2}\right) ; 66.7\left(\mathrm{CH}_{2}=\mathrm{CHCH}_{2} \mathrm{NCH}_{2} \mathrm{tBu}\right) ; 73.9\left(\mathrm{CH}_{\mathrm{c}} \mathrm{NCH}_{2} \mathrm{BBu}\right)$; $116.4\left(\mathrm{CH}=\mathrm{CH}_{2}\right) ; 136.7\left(\mathrm{CH}=\mathrm{CH}_{2}\right)$. IR $\left(\mathrm{NaCl}, \mathrm{cm}^{-1}\right): v=2953,2866,2806,1643,1480,1466$, 1393, 1362, 917. MS (70 eV) m/z (\%): 252 (M+ 5); 195 (100); 152 (23); 140 (14); 126 (40); 96 
(41); 71 (15); 70 (29); 56 (24). Rf = 0.74, Hexane/EtOAc 1/1. Anal. Calcd for $\mathrm{C}_{16} \mathrm{H}_{32} \mathrm{~N}_{2}$ : C 76.13, H 12.78, N 11.10. Found: C 76.35, H 12.95, N 10.98.

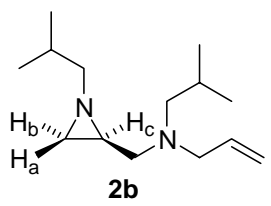

1-Isobutyl-2-( $N$-allyl- $\boldsymbol{N}$-(isobutyl)aminomethyl)aziridine (2b). Light-yellow oil. ${ }^{1} \mathrm{H}$ NMR (270 MHz, $\left.\mathrm{CDCl}_{3}\right): \delta$ 0.89, 0.93 and $0.96\left(12 \mathrm{H}, 3 \times \mathrm{d}, \mathrm{J}=6.6 \mathrm{~Hz}, 2 \times\left(\mathrm{CH}_{3}\right)_{2} \mathrm{CH}\right) ; 1.23(1 \mathrm{H}, \mathrm{d}, \mathrm{J}=6.6$ $\left.\mathrm{Hz}, \mathrm{H}_{\mathrm{b}}\right) ; 1.39-1.47\left(1 \mathrm{H}, \mathrm{m}, \mathrm{H}_{\mathrm{c}}\right)$; $1.55\left(1 \mathrm{H}, \mathrm{d}, \mathrm{J}=3.6 \mathrm{~Hz}, \mathrm{H}_{\mathrm{a}}\right) ; 1.70-1.89\left(2 \mathrm{H}, \mathrm{m}, 2 \times\left(\mathrm{CH}_{3}\right)_{2} \mathrm{CH}\right)$; 1.97-2.28 $\left(4 \mathrm{H}, \quad \mathrm{m}, 2 \times \mathrm{iPrCH}_{2} \mathrm{~N}\right) ; 2.35$ and $2.54(2 \mathrm{H}, 2 \times \mathrm{d} \times \mathrm{d}, \quad \mathrm{J}=13.4, \quad 5.7,5.7 \mathrm{~Hz}$, $\left.\left(\mathrm{H}_{\mathrm{a}} \mathrm{CH}_{\mathrm{b}}\right) \mathrm{CH}_{\mathrm{c}} \mathrm{CH}_{2} \mathrm{~N}\right)$; 3.11 and $3.17\left(2 \mathrm{H}, 2 \times \mathrm{d} \times \mathrm{d}, \mathrm{J}=14.4,6.6,6.6 \mathrm{~Hz}, \mathrm{~N}(\mathrm{HCH}) \mathrm{CH}=\mathrm{CH}_{2}\right)$; 5.08$5.19\left(2 \mathrm{H}, \mathrm{m}, \mathrm{CH}=\mathrm{CH}_{2}\right) ; 5.78-5.90\left(1 \mathrm{H}, \mathrm{m}, \mathrm{CH}=\mathrm{CH}_{2}\right) .{ }^{13} \mathrm{C} \mathrm{NMR}\left(68 \mathrm{MHz}, \mathrm{CDCl}_{3}\right): \delta 20.9,21.0$ and $21.1\left(2 \times\left(\mathrm{CH}_{3}\right)_{2} \mathrm{CH}\right)$; 26.5 and $29.2\left(2 \times\left(\mathrm{CH}_{3}\right)_{2} \mathrm{CH}\right)$; $33.4\left(\mathrm{H}_{\mathrm{a}} \mathrm{CH}_{\mathrm{b}}\right) ; 37.7\left(\mathrm{CH}_{\mathrm{c}}\right)$; 57.6 and 58.1 $\left(\left(\mathrm{H}_{\mathrm{a}} \mathrm{CH}_{\mathrm{b}}\right) \mathrm{CH}_{\mathrm{c}} \mathrm{CH}_{2} \mathrm{~N}\right.$ and $\left.\mathrm{NCH}_{2} \mathrm{CH}=\mathrm{CH}_{2}\right) ; 62.6$ and $69.5\left(2 \times \mathrm{iPrCH}_{2} \mathrm{~N}\right) ; 116.8\left(\mathrm{CH}=\mathrm{CH}_{2}\right) ; 136.4$ $\left(\mathrm{CH}=\mathrm{CH}_{2}\right)$. IR $\left(\mathrm{NaCl}, \mathrm{cm}^{-1}\right): v=1644,608 . \mathrm{MS}(70 \mathrm{eV}) \mathrm{m} / \mathrm{z}(\%): 224\left(\mathrm{M}^{+}, 13\right) ; 181(48) ; 152$ (17); 138 (63); 126 (60); 112 (100); 100 (19), 96 (47); 82 (22); 70 (91); 57 (28); 56 (34). Rf = 0.28, Hexane/EtOAc 1/1. Anal. Calcd for $\mathrm{C}_{14} \mathrm{H}_{28} \mathrm{~N}_{2}$ : C 74.94, $\mathrm{H}$ 12.58, N 12.48. Found: C 75.11, H 12.80, N 12.41.

1-(2,2-Dimethylpropyl)-2-pentylaziridine (3a). MS (70 eV) m/z (\%): 183 ( $\left.\mathrm{M}^{+}, 0.5\right) ; 182$ (1); 168 (12); 154 (6); 140 (8); 126 (100); 112 (84); 98 (11); 84 (46); 70 (29); 55 (33).

1-Isobutyl-2-pentylaziridine (3b) has been prepared as reported in the literature. ${ }^{3}$ Colorless

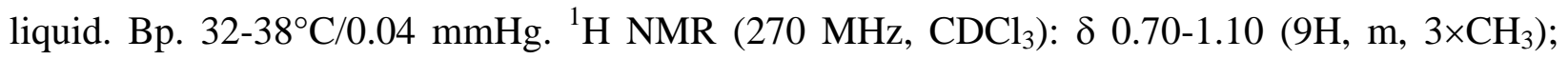
1.10-2.70 (14H, m, $\left.\mathrm{Me}_{2} \mathrm{CHCH}_{2} \mathrm{NCH}_{2} \mathrm{CH}\left(\mathrm{CH}_{2}\right)_{4}\right) .{ }^{13} \mathrm{C} \mathrm{NMR}\left(68 \mathrm{MHz}, \mathrm{CDCl}_{3}\right): \delta 14.1\left(\mathrm{CH}_{3} \mathrm{CH}_{2}\right)$; 21.0 and $21.1\left(\left(\mathrm{CH}_{3}\right)_{2}\right) ; 22.7\left(\mathrm{CH}_{2} \mathrm{CH}_{3}\right) ; 27.2\left(\mathrm{CH}_{2} \mathrm{CH}_{2} \mathrm{CH}_{2} \mathrm{CH}_{3}\right) ; 29.3\left(\mathrm{CHMe}_{2}\right) ; 31.8$ $\left(\mathrm{NCH}_{2} \mathrm{CH}\right)$; 33.1 and $34.1\left(2 \times \mathrm{CH}_{2}\right) ; 39.7\left(\mathrm{NCH}_{2} \mathrm{CH}\right) ; 69.9\left(\mathrm{NCH}_{2} \mathrm{iPr}\right) . \mathrm{IR}\left(\mathrm{NaCl}, \mathrm{cm}^{-1}\right): \mathrm{v}=1468$, 1383, 1363. MS (70 eV) m/z (\%): 169 (M+, 1); 140 (12); 126 (100); 112 (15); 98 (51); 84 (28); 71 (12); 70 (51); 58 (15); 57 (59); 56 (31); 55 (38); 43 (15); 42 (46); 41 (37). Anal. Calcd for $\mathrm{C}_{11} \mathrm{H}_{23} \mathrm{~N}$ : C 78.03, H 13.69, N 8.27. Found: C 78.17, H 13.88, N 8.39.

$\boldsymbol{N}$-Allyl- $\boldsymbol{N}$-butyl- $\boldsymbol{N}$-(2,2-dimethylpropyl)amine (4a). Isolated by means of preparative gas chromatography. ${ }^{1} \mathrm{H}$ NMR (270 $\left.\mathrm{MHz}, \mathrm{CDCl}_{3}\right): \delta 0.87\left(9 \mathrm{H}, \mathrm{s},\left(\mathrm{CH}_{3}\right)_{3} \mathrm{C}\right) ; 0.89-0.91(3 \mathrm{H}, \mathrm{m}$, $\left.\mathrm{CH}_{3} \mathrm{CH}_{2}\right) ; 1.10-1.60\left(4 \mathrm{H}, \mathrm{m},\left(\mathrm{CH}_{2}\right)_{2}\right) ; 2.17(2 \mathrm{H}, \mathrm{s}, \mathrm{NCH} \mathrm{tBu}) ; 2.47\left(2 \mathrm{H}, \mathrm{t}, \mathrm{J}=7.5 \mathrm{~Hz}, \mathrm{CH}_{2} \mathrm{CH}_{2} \mathrm{~N}\right)$; $3.12\left(2 \mathrm{H}, \mathrm{d} \times \mathrm{m}, \mathrm{J}=6.0 \mathrm{~Hz}, \mathrm{NCH}_{2} \mathrm{CH}=\mathrm{CH}_{2}\right) ; 4.9-6.3\left(3 \mathrm{H}, \mathrm{m}, \mathrm{CH}=\mathrm{CH}_{2}\right) .{ }^{13} \mathrm{C} \mathrm{NMR}(68 \mathrm{MHz}$, $\left.\left.\left.\mathrm{CDCl}_{3}\right): \delta 14.1\left(\mathrm{CH}_{3} \mathrm{CH}_{2}\right) ; 20.7\left(\mathrm{CH}_{3} \mathrm{CH}_{2}\right) ; 28.2\left(\mathrm{CH}_{3}\right)_{3} \mathrm{C}\right) ; 30.2\left(\mathrm{CH}_{2} \mathrm{CH}_{2} \mathrm{~N}\right) ; 33.1\left(\mathrm{CH}_{3}\right)_{3} \mathrm{C}\right)$; 56.5, 59.9 and $67.2\left(3 \times \mathrm{NCH}_{2}\right) ; 115.9\left(\mathrm{CH}_{2}=\mathrm{CH}\right) ; 137.1\left(\mathrm{CH}_{2}=\mathrm{CH}\right)$. IR $\left(\mathrm{NaCl}, \mathrm{cm}^{-1}\right): v_{\mathrm{C}=\mathrm{C}}=$ 1646. MS (70 eV) m/z (\%): 183 (M+, 4); 168 (5); 126 (100); 84 (29); 70 (17); 87 (6); 55 (5). Anal. Calcd for $\mathrm{C}_{12} \mathrm{H}_{25} \mathrm{~N}$ : C 78.62, H 13.74, N 7.64. Found: C 78.89, H 13.91, N 7.50.

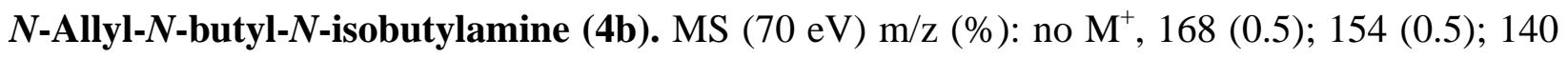
(0.5); 126 (100); 112 (17); 70 (7); 56 (3); 41 (21). 
$N$-(2,2-dimethylpropyl)- $N$-pentylamine (5a). MS (70 eV) m/z (\%): 157 (M+1 7); 142 (19); 100 (100); 86 (2); 84 (3); 72 (4); 71 (5); 70 (5); 57 (3); 56 (4); 55 (4).

$\boldsymbol{N}$-Isobutyl- $\boldsymbol{N}$-pentylamine (5b). Isolated by means of preparative gas chromatography. ${ }^{1} \mathrm{H}$ NMR (270 MHz, CDCl $): \delta 0.88\left(6 \mathrm{H}, \mathrm{d}, \mathrm{J}=6.0 \mathrm{~Hz},\left(\mathrm{CH}_{3}\right)_{2} \mathrm{CH}\right) ; 0.80-2.80\left(10 \mathrm{H}, \mathrm{m},\left(\mathrm{CH}_{3}\right)_{2} \mathrm{CH}\right.$ and $\left.\mathrm{CH}_{3}\left(\mathrm{CH}_{2}\right)_{3}\right) ; 2.43\left(2 \mathrm{H}, \mathrm{d}, \mathrm{J}=6.5 \mathrm{~Hz}, \mathrm{CHCH}_{2} \mathrm{~N}\right) ; 2.58\left(2 \mathrm{H}, \mathrm{m}, \mathrm{CH}_{2} \mathrm{CH}_{2} \mathrm{~N}\right) .{ }^{13} \mathrm{C} \mathrm{NMR}(68$ $\left.\mathrm{MHz}, \mathrm{CDCl}_{3}\right): \delta 14.0\left(\mathrm{CH}_{3} \mathrm{CH}_{2}\right) ; 20.7\left(\left(\mathrm{CH}_{3}\right)_{2} \mathrm{CH}\right) ; 22.7,29.7$ and $30.0\left(\mathrm{CH}_{3}\left(\mathrm{CH}_{2}\right)_{3}\right) ; 28.4$ $\left(\left(\mathrm{CH}_{3}\right)_{2} \mathrm{CH}\right) ; 50.3$ and $58.3\left(2 \times \mathrm{NCH}_{2}\right)$. IR $\left(\mathrm{NaCl}, \mathrm{cm}^{-1}\right): v_{\mathrm{NH}}=3310$. MS $(70 \mathrm{eV}) \mathrm{m} / \mathrm{z}(\%): 143$ $\left(\mathrm{M}^{+}, 6\right) ; 100$ (60); 86 (87); 57 (12).

Synthesis of $\mathbf{N}$-(2,2-dimethylpropyl)allylamine (6a). To a stirred solution of 2,2dimethylpropanal (pivaldehyde) 8a (2.15 g, 25 mmol) and anhydrous $\mathrm{MgSO}_{4}$ (4.51 g, 1.5 equiv) in dry dichloromethane $(25 \mathrm{~mL})$ was added allylamine $(1.51 \mathrm{~g}, 1.05$ equiv) at room temperature, and the resulting mixture was stirred for 1 hour under reflux. Filtration of the cooled reaction mixture and removal of the solvent in vacuo afforded $\mathrm{N}$-(2,2-dimethylpropylidene)allylamine 9 (3.01 g, 96\%). Subsequently, the latter aldimine 9 was dissolved in methanol (75 mL), and sodium borohydride ( $1.90 \mathrm{~g}, 2$ equiv) was added in small portions at $0^{\circ} \mathrm{C}$, followed by a reflux period of one hour. The reaction mixture was poured in water $(25 \mathrm{~mL})$, extracted with dichloromethane $(3 \times 20 \mathrm{~mL})$ and dried $\left(\mathrm{MgSO}_{4}\right)$. Filtration of the drying agent and removal of the solvent yielded $\quad N$-(2,2-dimethylpropyl)allylamine $\quad 6 a \quad(2.81 \quad$ g, $\quad 92 \%) . \quad N-(2,2-$ dimethylpropyl)allylamine (6a). ${ }^{1} \mathrm{H} \mathrm{NMR}\left(270 \mathrm{MHz}, \mathrm{CDCl}_{3}\right): \delta 0.95\left(9 \mathrm{H}, \mathrm{s},\left(\mathrm{CH}_{3}\right)_{3} \mathrm{C}\right) ; 1.20$ $(1 \mathrm{H}, \mathrm{s}(\mathrm{br}), \mathrm{NH}) ; 2.40\left(2 \mathrm{H}, \mathrm{s}, \mathrm{NCH}_{2} \mathrm{tBu}\right) ; 3.30\left(2 \mathrm{H}, \mathrm{d} \times \mathrm{m}, \mathrm{J}=6.0 \mathrm{~Hz}, \mathrm{NCH}_{2} \mathrm{CH}=\mathrm{CH}_{2}\right)$; 5.0-6.3 (3H, $\left.\left.\left.\mathrm{m}, \mathrm{CH}=\mathrm{CH}_{2}\right) .{ }^{13} \mathrm{C} \mathrm{NMR}\left(68 \mathrm{MHz}, \mathrm{CDCl}_{3}\right): \delta 27.9\left(\mathrm{CH}_{3}\right)_{3} \mathrm{C}\right) ; 31.5\left(\mathrm{CH}_{3}\right)_{3} \mathrm{C}\right) ; 53.5$ and 61.9 $\left(2 \times \mathrm{NCH}_{2}\right) ; 115.3\left(\mathrm{CH}_{2}=\mathrm{CH}\right) ; 137.6\left(\mathrm{CH}_{2}=\mathrm{CH}\right) . \mathrm{IR}\left(\mathrm{NaCl}, \mathrm{cm}^{-1}\right): v_{\mathrm{C}=\mathrm{C}}=1648 . \mathrm{MS}(70 \mathrm{eV}) \mathrm{m} / \mathrm{z}$ (\%): $127\left(\mathrm{M}^{+}, 4\right) ; 112$ (6); 70 (100); 68 (4); 56 (4).

$N$-Allyl- $N$-isobutylamine (6b). MS (70 eV) m/z (\%): 113 (M+1 7); 98 (1); 70 (100); 56 (4); 41 (33).

\section{Acknowledgements}

The authors are indebted to the "Fund for Scientific Research - Flanders (Belgium)" (F.W.O.Vlaanderen) and to Ghent University (GOA) for financial support.

\section{References}

1. (a) Lindström, U. M.; Somfai, P. Synthesis 1998, 109. (b) Zwanenburg, B.; ten Holte, P. Top. Curr. Chem. 2001, 94. (c) Sweeney, J. B. Chem. Soc. Rev. 2002, 31, 247. (d) Hu, X. E. Tetrahedron 2004, 60, 2701. (e) Tanner, D. Angew. Chem. Int. Ed. 1994, 33, 599. (f) Osborn, H. M. I.; Sweeney, J. Tetrahedron: Asymmetry 1997, 8, 1693. (g) MCoull, W.; Davis, F. A. Synthesis 2000, 1347. (h) Padwa, A.; Murphree, S. S. Arkivoc 2006, (iii), 6. 
2. (a) D'hooghe, M.; Waterinckx, A.; De Kimpe, N. J. Org. Chem. 2005, 70, 227. (b) D’hooghe, M.; Van Brabandt, W.; De Kimpe, N. J. Org. Chem. 2004, 69, 2703. (c) D’hooghe, M.; Van Speybroeck, V.; Waroquier, M.; De Kimpe, N. Chem. Commun. 2006, 1554. (d) D’hooghe, M.; Vanlangendonck, T.; Törnroos, K. W.; De Kimpe, N. J. Org. Chem. 2006, 71, 4678. (e) D’hooghe, M.; Van Speybroeck, V.; Van Nieuwenhove, A.; Waroquier, M.; De Kimpe, N. J. Org. Chem. 2007, 72, 4733.

3. D’hooghe, M.; Rottiers, M.; Jolie, R.; De Kimpe, N. Synlett 2005, 931.

4. (a) D’hooghe, M.; De Kimpe, N. Synlett 2004, 271. (b) D’hooghe, M.; Waterinckx, A.; Vanlangendonck, T.; De Kimpe, N. Tetrahedron 2006, 62, 2295. (c) D’hooghe, M.; De Kimpe, N. Synlett 2006, 2089. (d) D’hooghe, M.; De Kimpe, N. Arkivoc 2008, (ix), 6.

5. D’hooghe, M.; De Kimpe, N. Chem. Commun. 2007, 1275.

6. (a) Tayama, E.; Sugai, S. Synlett 2006, 849. (b) Bailey, W. F.; Luderer, M. R.; Jordan, K. P. J. Org. Chem. 2006, 71, 2825. (c) Christopher, A.; Brandes, D.; Kelly, S.; Minehan, T. Org. Lett. 2006, 8, 451.

7. (a) De Kimpe, N.; De Smaele, D.; Bogaert, P. Synlett 1994, 287. (b) De Kimpe, N.; Jolie, R.; De Smaele, D. J. Chem. Soc., Chem. Commun. 1994, 1221.

8. Flynn, D. L.; Zabrowski, D. L. J. Org. Chem. 1990, 55, 3673.

9. (a) Tidwell, T. T. Part 1, p 65 In The Chemistry of the Cyclopropyl Group, Wiley: N.Y., 1987. (b) Charton, M. In The Chemistry of Alkenes, Vol 2, p 511, Wiley: N.Y., 1970.

10. (a) Opitz, G.; Hellmann, H.; Schubert, H. W. Ann. 1959, 623, 112. (b) Chang, P. L.-F.; Dittmer, D. C. J. Org. Chem. 1969, 34, 2791. (c) Hall, H. K. Jr., Abdelkader, M.; Glogowski, M. E. J. Org. Chem. 1982, 47, 3691. 